\title{
Development of a cognitive screening instrument for tribal elderly population of Himalayan region in northern India
}

\author{
Sunil Kumar Raina, Sujeet Raina ${ }^{1}$, Vishav Chander, Ashoo Grover ${ }^{2}$, Sukhjit Singh ${ }^{3}$, Ashok Bhardwaj \\ Departments of Community Medicine, ${ }^{1}$ Medicine, ${ }^{3}$ Psychiatry, Dr. R.P. Government Medical College, Tanda, Himachal Pradesh, \\ ${ }^{2}$ Scientist, Indian Council of Medical Research (ICMR), New Delhi, India
}

\begin{abstract}
Introduction: Cognitive impairment, characteristic of dementia, is measured objectively by standard neuropsychological (cognitive) tests. Given the diversity of culture and language in India, it is difficult to use a single modified version of MMSE uniformly to Indian population. In this article, we report methods on the development of a cognitive screening instrument suitable for the tribal (Bharmour) elderly (6o years and above) population of Himachal Pradesh, India. Materials and Methods: We used a systematic, item-by-item, process for development of a modified version of MMSE suitable for elderly tribal population. Results: The modifications made in the English version of MMSE and the pretesting and pilot testing thereof resulted in the development of Bharmouri version of cognitive scale. Discussion: The study shows that effective modifications can be made to existing tests that require reading and writing; and that culturally sensitive modifications can be made to render the test meaningful and relevant, while still tapping the appropriate cognitive domains.
\end{abstract}

Key words: Cognitive, dementia, elderly, tribal, scale

\section{Introduction}

Cognitive impairment, characteristic of dementia, is measured objectively by standard neuropsychological (cognitive) tests. Screening for dementia is usually accomplished by means of a global cognitive scale, such as the widely used Mini-Mental State Exam (MMSE). ${ }^{[1,2]}$ This scale draws its strength from the fact that it briefly taps a variety of cognitive functions known to be impaired in dementia. ${ }^{[3]}$ The MMSE, modified and translated into Hindi has been previously used in the assessment of dementia in elderly population in India. ${ }^{[3]}$ This study conducted on Hindi knowing elderly population in northern part of India showed that the Hindi version was useful for assessing the cognitive functioning of study

\begin{tabular}{|l|l|}
\hline \multicolumn{2}{|c|}{ Access this article online } \\
\hline Quick Response Code: & Website: \\
\hline & www.ruralneuropractice.com \\
\cline { 2 - 3 } & \\
\hline
\end{tabular}

population. ${ }^{[3]}$ Systematic, item-by-item, empirically based test development has shown that effective modifications can be made to existing tests that require reading and writing; and that culturally sensitive modifications can be made to render the test meaningful and relevant while still tapping the appropriate cognitive domains. ${ }^{[3]}$ But given the diversity of culture and language in India, it is difficult to use a single modified version of MMSE uniformly to Indian population, so modifications suited to the study population are required.

Kashmiri and Dogri adaptation of the Mini-Mental State Examination (MMSE) are an example of the difficulty in developing a uniform modified version for Indian population. Those adaptations were required for use in two population groups living within the geographical limits of same district. ${ }^{[4,5]}$

Bharmour located between $32^{\circ} 11^{\prime}$ and $32^{\circ} 41^{\prime} \mathrm{N}$ latitude and $76^{\circ} 22^{\prime}$ and $76^{\circ} 53^{\prime} \mathrm{E}$ longitude is one of the notified tribal areas in Chamba district of the northwestern part of Himachal Pradesh [Figure 1]. Spread over an area of approximately $1818 \mathrm{sq}$. km, Bharmour has a mountainous topography having the altitudinal variation from

Address for correspondence:

Dr. Sunil Kumar Raina, Department Of Community Medicine, Dr. R.P. Government Medical College, Tanda, Himachal Pradesh, India. E-mail: ojasrainasunil@yahoo.co.in 


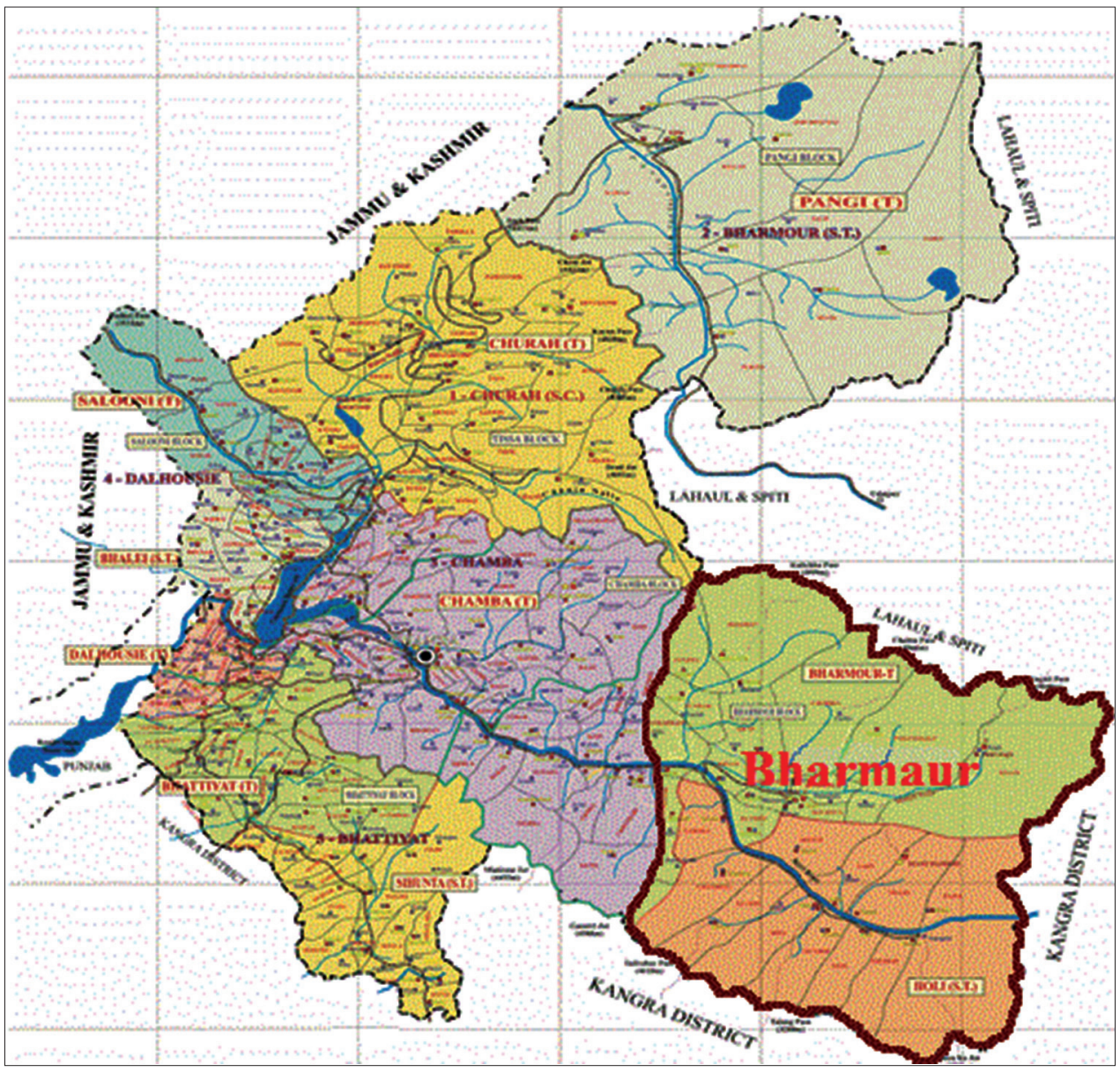

Figure 1: Map of Himachal Pradesh Showing the study area (Bharmour)

$1350 \mathrm{~m}$ to $5935 \mathrm{~m}$ above mean sea level. Bharmour area is characterized by a low resource base and fragile ecosystem and mainly inhabited by the Gaddi tribal community of only 39,113 persons (Male: 20,054 and Female: 19,059) (Census of India 2011) ${ }^{[6]}$ Bharmour is still one of the less developed regions in the state because of very unfavorable climate, peculiarly cold and dry, poor geographical accessibility and lack of basic infrastructural facilities. The majority of elderly individuals in this community has had no formal education and is illiterate. ${ }^{[7]}$

Bharmour elderly population being exclusively Bharmouri-speaking, its cognitive screening is a particular challenge. The development of a Bharmouri cognitive screening instrument suitable for the Bharmour elderly population is needed to overcome language and cultural issues specific to this cohort.

\section{Materials and Methods}

\section{Phases of instrument development \\ Initial work-up}

An expert group comprising of one bilingual investigator, two public health specialists, one internist and a psychiatrist from Himachal Pradesh developed for the Bharmour population, a series of measures that included the same general cognitive domains, as similar as possible to English version of MMSE in a language suited to the local Bharmouri population. This methodology was different to the one used by the principal investigator (one of the expert group members) earlier. ${ }^{[4,5]}$ The thought of comparing responses/scores in English or Hindi version versus local version did appear on our minds, but the thought was discarded considering the largely illiterate population of Bharmour.

The expert group sat together and worked on the possible alternatives to the English version of MMSE. An effort was made to arrive at consensus in development of questionnaire and a question was accepted for inclusion, if accepted upon by all expert group members.

\section{Background of expert group members Principal investigator (SKR)}

A public health specialist who has already worked on the development of Kashmiri and Dogri version of MMSE. ${ }^{[4,5]}$ Co-Investigators: AKB (second public health specialist) has been working in his area of expertise for last 25 years. 
SR (the third expert group, an internist) has already worked on a similar study on Kashmiri migrants. ${ }^{[4]}$ SS (Fourth expert group member, a Psychiatrist) has been working in his area of expertise for last 15 years. The bilingual investigator, a science graduate by qualification, has been working in the field of education as teacher for last 5 years.

\section{Pre-pilot and questionnaire layout}

The pre-pilot centered on information-gathering on the suitability of the questionnaire developed. For this purpose, we sat down with 25 subjects of age 60 years and above and went through the questions together to identify potential problems in the applicability of the new version of MMSE on the Bharmouri cohort. After a pre-piloting session, we amended the questionnaire before piloting with another group of 25 testers. The amended version was again based on unanimous decision by the expert group. This process was aimed at arriving at a scale wherein questions were unambiguous, appropriate and acceptable to respondents. We also tested the layout at this stage to ensure that field investigator can navigate their way easily through the questionnaire.

\section{Pilot phase}

After the appropriate modifications were made to the test items, an age-stratified (60-64, 65-69, 70-74, 75+) random sample of 50 non-demented subjects (as indicated clinically) was drawn from the Bharmour census database for a pilot study. The subjects were selected by simple random technique. After starting from the central part of Bharmour (bus stand area), one of the lanes was selected randomly by the lottery method using a currency note. Subjects were approached in their homes starting from the left side of the lane (after the toss of a coin in choosing the sides of lane). The process was repeated in similar manner in other lanes till a desired sample size of 50 was achieved.

The clinical assessment of dementia involved a careful detailed clinical history to determine the precise features of intellectual loss if any. The subjects were examined for three categories of symptoms: 1) cognitive or intellectual, 2) functional, and 3) psychiatric or behavioral. Field workers administered the cognitive battery (the modified version of MMSE), as finalized at the end of the pretest phase, to the subjects after obtaining informed consent and basic demographic information. The mean score obtained on modified version of MMSE on these 50 individuals was $28.77+1.04$. Each subject was assessed individually using the modified version of MMSE. The minimum time taken for assessing a single individual was $30 \mathrm{~min}$. The assessment included a brief on the purpose of the study and its prospective outcome. No one refused to participate in the study.

The demographic data and the mean score along with the standard deviation for age-stratified (60-64, 65-69, $70-74,75+)$ random sample of 50 non-demented subjects has been provided in detail in Table 1.

\section{Results}

The modifications made in the English version of MMSE and the pretesting and pilot testing thereof resulted in the development of Bharmouri version of MMSE, hereafter referred to as BMSE. The BMSE comprised of following test items [Table 2].

\section{Orientation to time}

In the English MMSE, a point each is given for correctly named day, date, month, year and season, for a total of five points. In our conversation with elderly population of Bharmour to explore feasibility, we found that elderly Bharmour residents did not appear to know or keep track of time in the Roman calendar but were quite conversant with day, date, month, and season in the Hindu calendar. However, they were not quite conversant with the concept of "year" in Hindu calendar.

The "year" was omitted in the modified version. A point each is given for correctly named time of day (morning, midday, evening, night), day, date, month, and season (cold, rainy and hot), for a total of five points.

\section{Orientation to place}

In English version of MMSE, five questions were asked, which could include 'name of this place/building', floor (storey), street address, city, county, state and country. It was difficult to translate them into Bharmouri for identification of spatial orientation. Villages in

Table 1: Demographic details and mini-mental state exam score of study population in pilot phase

\begin{tabular}{|c|c|c|c|c|c|c|}
\hline \multirow[t]{2}{*}{ Age group } & \multicolumn{3}{|c|}{$n(\%)$} & \multicolumn{3}{|c|}{ MMSE score (Mean \pm SD) } \\
\hline & Male (\%) & Female (\%) & Total (\%) & Male & Female & Total \\
\hline $60-64$ years & $8(16.0)$ & $6(12.0)$ & $14(28.0)$ & $29.38 \pm 0.52$ & $29.17 \pm 0.40$ & $29.29 \pm 0.46$ \\
\hline $65-69$ years & $7(14.0)$ & $5(10.0)$ & $12(24.0)$ & $29.29 \pm 0.48$ & $29.20 \pm 0.84$ & $29.25 \pm 0.62$ \\
\hline $70-74$ years & $5(10.0)$ & $8(16.0)$ & $13(26.0)$ & $29.00 \pm 0.00$ & $29.00 \pm 0.53$ & $29.00 \pm 0.40$ \\
\hline 75 years and above & $6(12.0)$ & $5(10.0)$ & $11(22.0)$ & $26.83 \pm 0.75$ & $27.80 \pm 1.30$ & $27.27 \pm 1.10$ \\
\hline Total & $26(52.0)$ & $24(48.0)$ & $50(100.0)$ & $28.69 \pm 1.16$ & $28.83 \pm 0.92$ & $28.77 \pm 1.04$ \\
\hline
\end{tabular}


Raina, et al.: Cognitive screening instrument for tribal elderly

Table 2: Showing two versions of mini-mental state exam

\begin{tabular}{lll}
\hline $\begin{array}{l}\text { Max. } \\
\text { score }\end{array}$ & MMSE (english version) & MMSE (bharmour version) \\
\hline 5 & $\begin{array}{l}\text { "What is the year? Season, date, day of the week } \\
\text { and month?" }\end{array}$ & "What is the time of day? Day, date, month, and season \\
5 & $\begin{array}{l}\text { "Where are we now: State/County/Town/city/ } \\
\text { Hospital/Floor }\end{array}$ & "Where are we now: Country, state, District, block, Panchayat, Village
\end{tabular}

3 The examiner names three unrelated objects clearly and slowly, then asks the patient to name all three of them. The patient's response is used for scoring. The examiner repeats them until patient learns all of them, if possible. Number of trials:

$5 \quad$ I would like you to count backward from 100 by sevens." $(93,86,79,72,65, \ldots)$ Stop after five answers

Alternative: "Spell WORLD backwards." (D-L-R-O-W)
The examiner names three unrelated objects in Bharmouri language clearly and slowly, then asks the patient to name all three of them. The patient's response is used for scoring. The examiner repeats them until patient learns all of them, if possible. Number of trials:

We constructed a story for the subtraction task: 'You went to a grocer with a fifty rupees note to make a purchase of seven rupees. After paying him how much will be left with you? In the next morning you went again to purchase the same item, how much will be left with you?" The first five consecutive responses are scored

World backwards: Since most of the sample is illiterate, spelling (either forwards or backwards) is not an option. We ask subjects to name the months in Hindu calendar backwards, starting from the current month. Responses were scored for the 5 months named, not including current month. This was thought as closest possible to "world backwards" option in capturing attention of the subjects

3 "Earlier I told you the names of three things. Can you tell me what those were?

2 Show the patient two simple objects, such as a wristwatch and a pencil, and ask the patient to name them

1 Repeat the phrase: 'No ifs, ands, or buts

3 Take the paper in your right hand, fold it in half, and put it on the floor." (The examiner gives the patient a piece of blank paper.)

$1 \quad$ "Please read this and do what it says." (Written instruction is "Close your eyes.")

1 "Make up and write a sentence about anything." (This sentence must contain a noun and a verb.)

$1 \quad$ "Please copy this picture." (The examiner gives the patient a blank piece of paper and asks him/her to draw the symbol below. All 10 angles must be present and two must intersect.)

Earlier I told you the names of three things. Can you tell me what those were?

Show the patient two simple objects, such as a wristwatch and a pencil, and ask the patient to name them

Repeat the phrase with: now, when, then as focus

Take the paper in your right hand, fold it in half, and put it on the floor." (The examiner gives the patient a piece of blank paper.)

The examiner says 'just see what I am doing and repeat the same" and then closes his own eyes for 3 seconds (follow example) and later examiner observes the subject. (See and follow command)

A meaningful sentence generation in response to the question by the examiner

Please copy this picture." (The examiner gives the patient a blank piece of paper and asks him/her to draw the symbol below

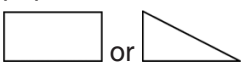

$30 \quad$ Total

MMSE: Mini-mental state exam

Bharmour do not have street numbers; streets do not have names.

However, villagers in Bharmour are quite familiar with the administrative hierarchy of the state of Himachal Pradesh. Therefore, a new subset of questions registering orientation to place was developed in modified version keeping this knowledge in view. Modified test items enquired about country, state, district/block, Panchayat (village level administrative unit consisting of a few villages) and village. A point each is awarded for correctly answering each.

\section{Registration}

In the English MMSE, the names of three objects are given. We modified the initial objects in English version with three common objects used in Bharmour. We have used three Bharmouri words, most commonly used in Bharmour language for apple, rupee and bed. The subjects were later asked to recall the three objects.

\section{Attention and calculation}

The MMSE has two alternative attention subtests: (i) serial subtractions of seven starting at 100. (ii) Backwards spelling of WORLD and Serial sevens.

We constructed a story for the subtraction task:

"You went to a grocer with a fifty rupees note to make a purchase of seven rupees. After paying him how much will be left with you? In the next morning you went again to purchase the same item, how much will be left with you?" The first five consecutive responses are scored. 


\section{Backwards spelling of WORLD}

Since most of the sample is illiterate, spelling (either forwards or backwards) is not an option. We ask subjects to name the months in Hindu calendar backwards, starting from the current month. Responses were scored for the 5 months named, not including current month. This was thought as closest possible to "world backwards" option in capturing attention of the subjects.

\section{Recall}

The subject is asked to recall the three objects named earlier.

\section{Naming}

We retained the questions used in English MMSE, as they were feasible in our study population in pre-pilot phase/pilot phase of testing.

\section{Repetition}

We modified this question by replacing it with a phrase similar to the standard phrase in English version, but quite common in Bharmour language. The modified version revolved around three words, now, when and then.

\section{Three-step task}

We retained the questions used in English MMSE, as they were feasible in our study population in pre-pilot phase/pilot phase of testing.

\section{Visual command ()}

We substituted this subtest with a see and follow command instead of read and follow command of the English version. The examiner says 'just see what I am doing and repeat the same" and then closes his own eyes for 3 seconds (follow example) and later the examiner observes the subject.

\section{Sentence}

This task is not practical for our study population due to literacy reasons, so we replaced it with the capacity of individuals to complete a meaningful sentence in response to a specific question asked by the investigator. We attempted to record the capacity of elderly to understand the task of generating a complete thought. The question was based on housing, livestock or agriculture, like in case of a flood in your area, what will happen to your house, livestock or agriculture?. A task was accepted as complete only if a meaningful sentence was generated in response to the question by the village elders.

\section{Copying a figure}

We replaced copying intersecting pentagons and instead awarded any diagram involving an intersection of any two geometrical figures. This was because we realized the inability of the study population in drawing a pentagon correctly as they seemed to have no concept of geometrical figures. For this reason, intersecting triangles or intersecting rectangles was taken as correct. The inability of being absolutely precise in formation of angles was not taken into account while awarding marks. This subtest is an example of why clinical judgment is required for scoring, as opposed to administration and recording, of responses.

\section{Discussion}

Till recently, the consensus figure for prevalence of dementia in India was used from the paper, Global prevalence of dementia: a Delphi consensus study. ${ }^{[8]}$ This paper gives the 2001 dementia population of India as 1.5 million. According to the paper, this is set to increase by more than $300 \%$ by 2040 (that is, it is estimated to reach six million).The Dementia India Report 2010 released in September 2010 estimates the prevalence as higher than those estimated by the above Delphi consensus. ${ }^{[9]}$ According to the report: For the year 2010, an estimated 3.7 million India people aged over 60 have dementia (2.1 million women and 1.5 million men).The report points out that Indian studies of dementia have not been uniform across the country, with six studies from the Southern region and one each from the West, East, and Northern region. There has been a wide variation in the estimates, making it difficult to provide a consistent view for the entire country. This may partly be because of absence of a uniform measurement scale throughout the country.

Although, MMSE is among the most widely used of general mental status tests; it is important that attention is paid to the appropriateness of each of its subtests and items for the population being studied. This probably necessitates a modification in the subtests used in English version to suit to the local population for assessment of prevalence of dementia.

The present study is a part of the study to arrive at prevalence of dementia in selected elderly population of Himachal Pradesh. One of the populations selected for the purpose of study is a tribal population located in Bharmour area of Himachal Pradesh. The assessment of dementia in this population necessitated developing of a scale suited to the largely illiterate, Bharmouri speaking population. For this purpose we used the English version of MMSE as the base and made modification suited to our population. The process was almost similar in intent to development of Hindi, Kashmiri and Dogri version of MMSE..$^{[3-5]}$

In our modification of the English version of MMSE, some subtests required substantial modification for 
Bharmour population, which clearly seemed to be related to education. The modifications included the attention subtest requiring abstract mental arithmetic skills for which this population has no other use. Modification was also necessitated in subtests which require reading, writing and drawing.

It was also observed that Orientation to time, in the conventional sense, does not appear to play a major role in the daily routine functioning of Bharmour elderly population. This is in line with an earlier study conducted on a rural elderly population in India. ${ }^{[3]}$

A growing body of epidemiological literature suggests that lower levels of education are associated with higher prevalence of dementia and lack of education may be a risk factor for Alzheimer's disease. ${ }^{[8,9]}$

If there is a genuine difference in risk, one would expect to find higher prevalence rates of Alzheimer's diseases in societies with lower educational levels, perhaps in pandemic proportions in subgroups with no formal education. ${ }^{[10,11]}$ While such a hypothesis requires study of vastly under educated societies, it simultaneously magnifies the methodological challenge of developing appropriate cognitive tests for undereducated and illiterate populations.

In this study, a multitude of cultural and linguistic factors of the original English MMSE were identified as being unfeasible to our study population. Examples of cultural irrelevance include interpretations of these subjects; with respect to season. Bharmouri individuals are more likely to interpret and describe the observable weather as season. This understanding is rooted in an existing relation between cultural practices and particular weather conditions interpreted as seasons.

This study highlighted the challenge in retaining the essential elements of each test item of the original MMSE while adapting the test to a specific regional cultural group such as the Bharmour.

The results in this study are drawn from age-stratified (60-64, 65-69, 70-74, 75+) random sample of 50 non-demented subjects (proven clinically) from Bharmour census database. The mean score obtained on modified version of MMSE on these 50 individuals was $28.77+1.04$. The concordance of the score with the clinical findings helped us in accepting the modified version of MMSE as a standard tool for screening dementia in elderly population in Bharmour. Future work in progress will address the utility of the Bharmouri Mental State Exam as a screening instrument for dementia in this population. Data on prevalence of dementia in Himachal Pradesh is sparse. This holds true for Bharmour too.

While the resulting instrument should be 'fair' to the populations being studied, it will not necessarily be equally fair for all populations, for example, it is Unfair to administer the Bharmouri version of MMSE to non-Bharmours; however, the systematic approach to instrument development should be adopted to investigations of other populations. As demonstrated in this study, similar modifications to the MMSE which are valid and reliable may be attempted for other remote regional populations.

\section{Conclusions}

While our modification of the MMSE will not be equally appropriate for screening another population, our methods of test development should be useful to others conducting similar studies, particularly those involving comparisons between diverse populations. The importance of this paper lies in its capacity to capture dementia in non-Hindi knowing tribal population.

\section{Limitations of the study}

In an ideal setting, the validity of screening test for dementia should have been confirmed by its ability to discriminate between samples of demented and non-demented individuals. It would have been relatively easy in a case-control study, which unfortunately was not the case in our study.

\section{References}

1. Folstein MF, Folstein SE, McHugh PR. Mini-Mental State: A practical method for grading the cognitive state of patients for the clinician. J Psychiatr Res 1975;12:189-98.

2. Tombaugh TN, McIntyre NJ. The Mini-Mental State Examination: A comprehensive review. J Am Geriatr Soc 1992;40:922-35.

3. Ganguli M, Ratcliff G, Chandra V, Sharma S, Gilby J, Pandav R, et al. Hindi version of the MMSE: The development of a cognitive screening instrument for a largely illiterate rural elderly population in India. Int J Geriatr Psychiatr 1995;10:367-77.

4. Raina S, Razdan S, Pandita KK, Raina S. Prevalence of dementia among Kashmiri migrants. Ann Indian Acad Neurol 2008;11:106-8.

5. Raina SK, Razdan S, Pandita KK. Prevalence of dementia in ethnic Dogra population of Jammu district, North India: A comparison survey. Neurol Asia 2010;15:65-6.

6. Himachal Pradesh-census of India website. Available from: http://www. censusindia.gov.in [Last accessed on 2012 Sep 15].

7. Tribal areas- Government of Himachal Pradesh. Available from: http://www.himachal.nic.in/tribal/tribalarea.htm [Last accessed on 2012 Sep 15].

8. Ferri CP, Prince M, Brayne C, Brodaty H, Fratiglioni L, Ganguli M, et al. Global prevalence of dementia: A Delphi consensus study. Lancet 2005;366:2112-7. 
9. The Dementia India Report 2010. Available from: http://www.alzheimer. org.in/assets/dementia.pdf [Last accessed on 2012 Sep 10].

10. Katzman R. Education and the prevalence of dementia and Alzheimer's disease. Neurology 1993;43:13-20.

11. Mortimer JA, Graves A. Education and other socio-economic determinants of dementia and Alzheimer's disease. Neurology 1993;43 (Suppl 4):39-44.
How to cite this article: Raina SK, Raina S, Chander V, Grover A, Singh S, Bhardwaj A. Development of a cognitive screening instrument for tribal elderly population of Himalayan region in northern India. $\mathrm{J}$ Neurosci Rural Pract 2013;4:147-53.

Source of Support: Indian Council of Medical Research. Conflict of Interest: None declared. 